\title{
EN PROCESO DE CONSTRUCCIÓN DEL CAPITAL RELACIONAL \\ Activos intangibles generadores de oportunidades de negocio
}

\author{
Mary Luz Ordoñez Santos*
}

\footnotetext{
* Estudiante del programa de Doctorado La Empresa en una Economía Internacionalizada, CEU Universidad San Pablo, Madrid, España. Especialista en Administración de Personal, EAN, 2004. Especialista en Cultura Política, 2001. Magister en Administración de Empresas, USTA, Bucaramanga, 1998. Administrador de Empresas, UFPS, 1993. Profesora Asistente adscrita a la Facultad de Ciencias Económicas de la Universidad de Pamplona. E-mail: ordonez_et_al@hotmail.com
} 


\section{EN PROCESO DE CONSTRUCCIÓN DEL CAPITAL RELACIONAL Activos intangibles generadores de oportunidades de negocio}

\section{RESUMEN}

El artículo proyecta presentar una propuesta sobre el proceso de construcción del capital relacional, herramienta en el modelo de gestión de activos intangibles orientada a que las empresas planifiquen actividades en función a sus relaciones con el entorno.

El trabajo se desarrolla en tres etapas, la primera consiste en abordar, desde la perspectiva financiera, la naturaleza del significado intangible; en la segunda etapa, desde la perspectiva de mercado intenta identificar el valor oculto del capital relacional, finalmente busca el punto de partida, que permita identificar los parámetros de construcción del capital relacional como generador de oportunidades de negocio.

Palabras Claves: Activos intangibles, administración del conocimiento, capital relacional.

\begin{abstract}
The projection of this article is to present a proposal about the process of construction with relational capital as a tool in the model of management for intangible active resources oriented to what business activities plan in the function to the relations within their sphere.

The work is developed in three stages: the first consists of confronting, from a financial perspective, the nature of the significant intangible; the second stage, from the market perspective, intends to identify the hidden value of the relational capital. Finally, returning to the point of departure, all of this permits us to identify the parameters of construction with that relational capital as the generator of enterprise opportunities.
\end{abstract}

Keywords: intangible assets, knowledge management, relational capital.

\section{Face IsSN 1794-9920}

Recepción: Julio de 2008

Revisión: Diciembre de 2008 Aceptación: Diciembre de 2008 


\section{PERSPECTIVA FINANCIERA}

\section{Naturaleza intangible}

Abordar el capital relacional desde el punto de vista financiero, entendido como la combinación de activos inmateriales o intangibles que permiten funcionar la empresa en un entorno, suena a una tarea compleja que conlleva inicialmente a identificarles y valorarlos adecuadamente.

En los últimos años, se viene exponiendo que el Capital Relacional se refiere al valor que genera para la empresa, las relaciones que mantiene con el exterior, comprendiendo las relaciones con clientes y proveedores.

Y es que el significado general del concepto intangible es la carencia de sustancia física, el Diccionario define a intangible como: "No tangible; incapaz de ser percibido por el tacto; impalpable; imperceptible".

El estudio de los intangibles, ha sido abordado de varios puntos de vista, ya sea como activos intangibles, activos de conocimiento, y capital intelectual, todos términos ampliamente divulgados. Activo intangible en la literatura contable, activos de conocimiento en la literatura económica, y capital intelectual en la literatura de dirección estratégica de empresas; pero ellos se refieren esencialmente a la misma cosa: características no físicas que generan beneficios económicos futuros para la empresa.

Se pudiera afirmar, con un alto grado de certeza que, el problema fundamental que se presenta en los intangibles es determinar propiamente lo que son los intangibles y cuáles son los que cumplen con los requisitos de ser considerados como activo.

No obstante, el Centro de Investigación de Intangibles de la Escuela Stern de la New York University, dirigido por el académico Baruch L., propone la siguiente definición de intangibles: "Fuentes de beneficios económicos, que carece de sustancia física o, alternativamente, como todos los elementos de una empresa que existen además de los activos tangibles y monetarios"

Esta definición que da la Escuela Stern de la New York University se divide en dos puntos:

a) Primero que establece cual intangible corresponde a "fuentes de beneficios económicos, que carecen de sustancia física". Esta definición es bastante amplia, en el sentido que corresponde al potencial para contribuir directa o indirectamente al flujo de efectivo o equivalente, este potencial de contribución puede ser productivo o de convertibilidad; productivo, ya que es parte de las operaciones operativas de la empresa y convertibilidad por su capacidad de transformarse en efectivo y efectivo equivalente. 
b) Segundo, la definición indica que el intangible se puede entender "...como todos los elementos de una empresa que existen además de los activos tangibles y monetarios". Esta parte de la definición indica que cualquier elemento que no sea activo tangible y/o monetario, es intangible.

La definición dada por la Escuela Stern es amplia, en el sentido que define el concepto de intangible, pero no explica cuestiones fundamentales tales como: el reconocimiento, la amortización, la duración.

La literatura tradicional ha clasificado a los activos en dos tipos: tangibles e intangibles. Tradicionalmente los activos tangibles (activos físico y financiero) constituían el activo más preciado de las empresas, sin embargo, en los últimos años del siglo XX esta idea dio paso al considerar a los activos intangibles como elementos claves para competir en entornos dinámicos.

Palomo, M. (2003) presenta el enfoque de Edvinsson y Malone (1998), hacia la clasificación de activos intangibles (cuadro No. 01).

\section{Cuadro No.01}

\begin{tabular}{|l|l|l|l|}
\hline \multicolumn{4}{|c|}{ Clasificación de activos intangibles } \\
\hline Marcas de servicios & $\begin{array}{c}\text { Activos de } \\
\text { propiedad } \\
\text { intelectual }\end{array}$ & \multicolumn{1}{|c|}{$\begin{array}{c}\text { Activos } \\
\text { Humanos }\end{array}$} & \multicolumn{1}{|c|}{$\begin{array}{c}\text { Activos de } \\
\text { infraestructura }\end{array}$} \\
\hline Marcas de productos & Patentes & Educación & Filosofía administrativa \\
\hline Marcas corporativas & Derechos de autor & Calificaciones & Cultura corporativa \\
\hline Clientes & $\begin{array}{l}\text { Conocimiento } \\
\text { sobre actividades }\end{array}$ & Procesos Administrativos \\
\hline Lealtad del consumidor & Saber-hacer & Competencias & $\begin{array}{l}\text { Sistemas de información } \\
\text { tecnológica }\end{array}$ \\
\hline Continuidad de negocios & Marcas de redes \\
\hline Nombre de la empresa & Marcas de servicios & $\begin{array}{l}\text { Evaluaciones de } \\
\text { puestos y } \\
\text { psicométricas }\end{array}$ & Relaciones financieras \\
\cline { 1 - 2 } Blacklog & \multicolumn{3}{|l|}{} \\
\hline
\end{tabular}

Es necesario señalar que un problema particular de los activos intangibles es su fragilidad, dado que ellos no pueden ser completamente apropiables por aquello que contribuyen a su creación (Benedetto, G. 1996).

En esta época, la gerencia debe conducir operaciones e inversiones rentables que permitan el crecimiento de la empresa. Por ello, la correcta gestión de los intangibles puede lograrse mediante el aprovechamiento de las oportunidades que brinda el medio, 
fortaleciendo el crecimiento que da solvencia y acrecientan el capital de trabajo para los nuevos ciclos de vida.

Los propósitos inherentes a la consolidación en el mercado, el incremento progresivo de los índices de productividad y eficiencia, la satisfacción de las necesidades cambiantes del consumidor o la conquista de capital relacional son una base sobre la que descansan la maximización del patrimonio.

$Y$ es que al referirnos al valor que tiene para la empresa el conjunto de relaciones que mantiene con el exterior; la competencia de interacción con el entorno y el mercado, permite la percepción de un elemento diferenciador sui géneris para respaldar sus actividades.

De igual manera, quienes tienen responsabilidades financieras en cualquier organización saben que sus decisiones deben responder a maximizar la generación de utilidades como fin de toda empresa, las cuales se encuentran afectadas por las decisiones de corto y largo plazo.

No en pocas ocasiones, la evaluación financiera que determina calificar el éxito o fracaso de una gestión gerencial, no puede centrarse de manera exclusiva en la información suministrada por la contabilidad convencional.

Obviamente la función financiera y la administrativa, deben incluir el propósito y la firme convicción de cumplir con los objetivos del negocio, dentro de un marco ético, moral y legal que le permita una rentabilidad justa y equitativa, cumpliendo los compromisos con los empleados, proveedores, propietarios, la sociedad y la economía en general.

\section{PERSPECTIVA DEL MERCADO}

\section{Valor oculto del capital relacional}

La connotación del modelo Technology Broker propuesto por A. Brooking (1996) clasifica los activos intangibles en cuatro categorías, las cuales justifican el concepto de capital intelectual. Debemos indicar que para Brooking estos activos son: los activos de propiedad intelectual, los activos humanos, los activos de infraestructura, los activos de Mercado.

Para la comprensión de los elementos básicos del enfoque de los activos de mercado, deberíamos revisar aquellos que se derivan de una relación beneficiosa de la empresa con su mercado y sus clientes y por tanto denotan propiedad e identidad de los productos y servicios que ofrece, al mismo tiempo que aseguran estrategias de posicionamiento que proporcionan una ventaja competitiva en el mercado y en 
EN PROCESO DE CONSTRUCCIÓN DEL CAPITAL RELACIONAL

Activos intangibles generadores de oportunidades de negocio

ocasiones son la causa de que algunas empresas se adquieran por sumas superiores a su valor contable.

Basándose en algunas interpretaciones de su obra, estos activos constituyen el potencial derivado de los bienes inmateriales que guardan relación con el mercado, sus indicadores son: marcas, clientes, cartera de pedidos, distribución, capacidad de colaboración, fidelidad de la clientela, canales de distribución y la razón social de la empresa entre otros.

Desde una perspectiva más pragmática, se observa que el capital relacional son los activos acumulados por la empresa gracias a sus relaciones con agentes de su entorno (Palacios, 2002); con una preocupación netamente técnica, en un principio se le denominaba capital cliente, luego se renombró y amplió el término, puesto que de esta forma podía incluir no sólo las relaciones con los clientes, sino también con asociaciones, competidores, proveedores, gobierno, etc. en definitiva, con el resto de agentes del entorno.

El pensamiento de capital cliente representa los conocimientos de la organización sobre aquellas relaciones que afectan a los clientes y que constituyen fuentes de ventajas competitivas (Onge, 1996). Es así como al estudiar el valor de las relaciones entabladas entre la empresa y la gente con la que hace sus negocios, se fundamenta en el valor procedente de las relaciones de la organización, lo que implica conocer bien al cliente.

Esta construcción, pareciera no haber ignorado el intercambio de información con externos; de ahí que el capital relacional estudia las relaciones de la organización con los agentes de su entorno, se refiere a la cartera de clientes, a las relaciones con los proveedores, bancos y accionistas, a los acuerdos de cooperación, de producción y comerciales, a las marcas comerciales y a la imagen de la empresa, medios de comunicación y alianzas estratégicas. La validez del planteamiento que, estos activos son propiedad de la empresa y algunos de ellos pueden protegerse legalmente, como es el caso de las marcas comerciales, en la realidad inmediata, al depender de una relación con terceros, no puede controlarse completamente por la organización.

La preocupación porque todos aquellos elementos y fuerzas, no tangibles, incluidos los conocimientos tácitos y explícitos (marcas, patentes, software, etc.), que dentro de un marco estratégico específico, conducen a la creación de valor de los activos físicos, tangibles, que inciden directamente en el valor agregado de las organizaciones, corresponde al capital que reside en la cabeza de las personas. El camino "ideal" en el sentido de lo deseable, sería tratar de convertir el conocimiento explícito de la organización en beneficios monetarios medibles. 
Si la innovación es una de las características del mundo moderno y su manifestación se da en la administración correcta de los activos intelectuales, esta no puede concebirse aisladamente, circunscritas únicamente a tener ventajas competitivas y un buen posicionamiento en el mercado, sentar las bases del buen desarrollo organizacional, tras un estudio previo de las características personales de los empleados al elaborar modelos para realizar la gestión y obtener ventajas substanciales a través de productos de un uso más directo del conocimiento pudiera ser más productivo.

Para estas circunstancias, la inteligencia competitiva será la observación y análisis del negocio (clientes, proveedores y, sobre todo, competidores), pero también del entorno social y económico que se traduce en los hábitos de consumo o en la legislación que puede cambiar la orientación del mercado; igualmente, está ejemplificada en la vigilancia tecnológica de las oportunidades y amenazas que para una empresa supone la innovación técnico-científica.

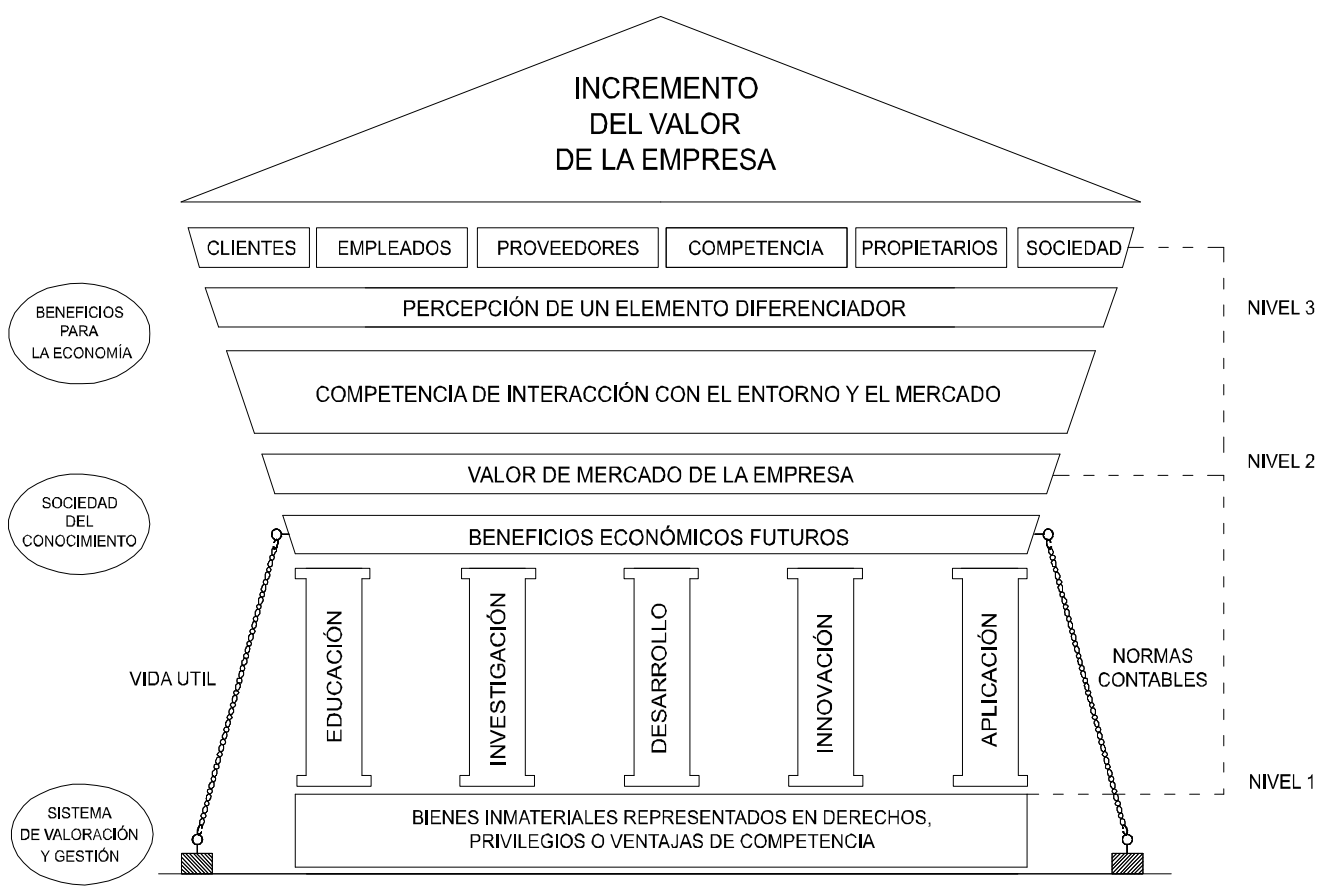

FUENTE: Elaboración propia 
Como se ha indicado previamente, por capital relacional se entiende el valor que tiene para la empresa el conjunto de relaciones que mantiene con el exterior, las relaciones con otros agentes externos (clientes, proveedores, gobierno...), el hecho que no exista un modelo estandarizado de medición, corresponde justamente a la forma como la administración comprende la diferencia entre el valor contable y el valor de mercado de una empresa.

Mientras los activos de mercado de una organización constituyen el potencial derivado de los bienes inmateriales que guardan relación con el mercado, tales como las marcas, los clientes y su fidelidad, el prestigio, los canales de distribución, contratos, acuerdos, licencias y franquicias, entre otros. Una vez este tipo de activos aseguran que los clientes conozcan la identidad de la empresa y lo que hace, el interés del planteamiento radica en que el capital relacional pueda ser definido como la habilidad de la empresa para interactuar positivamente con la comunidad empresarial y así estimular su potencial de creación de riqueza animando el capital humano.

\section{PROPUESTA DE DEFINICIÓN}

\section{Punto de partida: parámetros de construcción}

Como se citó anteriormente, no existe un modelo estandarizado de medición del capital intelectual de una organización, sin embargo, el análisis de éste se considera un recurso clave en la generación de una ventaja competitiva sostenible para la organización, encaminado a comprender la diferencia entre el valor contable y el valor de mercado de una empresa; como se indica en el cuadro 02, existen algunos modelos que buscan la identificación de dichos activos ocultos:

- Método Intelec: Estructurado en bloques, elementos e indicadores y dividen el capital estructural en capital tecnológico y capital organizativo.

- Intellectual Assets Monitor: Los activos intangibles se medirían según su orientación, bien fuera hacia el exterior (clientes, accionistas, proveedores...) o hacia el interior (directivos, empleados).

- Navigator de Skandia: conjunto de actividades intangibles, no reflejadas en las actividades tradicionales, pero que inciden en los futuros flujos de caja.

- Balanced Scorecard: Modelo que integra indicadores financieros (de pasado) y no financieros (de presente o futuro) 


\begin{tabular}{|c|c|c|}
\hline \multicolumn{3}{|c|}{ MODELOS Y HERRAMIENTAS DE CAPITAL INTELECTUAL } \\
\hline $\begin{array}{c}\text { MODELOS Y } \\
\text { HERRAMIENTAS }\end{array}$ & ESTRUCTURA & $\begin{array}{c}\text { CARACTERÍSTICAS } \\
\text { RELEVANTES }\end{array}$ \\
\hline $\begin{array}{l}\text { Navegador de Skandia } \\
\text { (Edvinsson } \\
\text { 1992-1997) }\end{array}$ & $\begin{array}{l}\text { - Enfoque financiero } \\
\text { - Enfoque cliente } \\
\text { - Enfoque humano } \\
\text { - Enfoque procesos } \\
\text { - Enfoque renovación }\end{array}$ & $\begin{array}{l}\text { - Indicadores de medida } \\
\text { absoluta } \\
\text { - Indicadores de eficiencia }\end{array}$ \\
\hline $\begin{array}{l}\text { University of Western Ontario } \\
\text { (Bontis, 1996) }\end{array}$ & $\begin{array}{l}\text { Relación de causalidad entre: } \\
\text { - Capital humano } \\
\text { - Capital relacional } \\
\text { - Capital organizativo }\end{array}$ & - Indicadores de resultados \\
\hline $\begin{array}{l}\text { Monitor de Activos } \\
\text { Intangibles } \\
\text { (Sveiby, 1997) }\end{array}$ & $\begin{array}{l}\text { - Estructura interna } \\
\text { - Estructura externa } \\
\text { - Competencias de los } \\
\text { empleados }\end{array}$ & $\begin{array}{l}\text { - Indicadores de crecimiento y } \\
\text { renovación } \\
\text { - Indicadores de eficiencia } \\
\text { - Indicadores de estabilidad }\end{array}$ \\
\hline $\begin{array}{l}\text { Modelo Intelect (Euroforum, } \\
\text { 1997- } \\
1998 \text { ) }\end{array}$ & $\begin{array}{l}\text { - Bloque de capital humano } \\
\text { - Bloque de capital estructural } \\
\text { - Bloque de capital relacional }\end{array}$ & $\begin{array}{l}\text { - Indicadores de presente y } \\
\text { futuro }\end{array}$ \\
\hline $\begin{array}{l}\text { Modelo Nova (Camisón, } \\
\text { Palacios y } \\
\text { Devece, 1998) }\end{array}$ & $\begin{array}{l}\text { - Capital humano } \\
\text { - Capital organizativo } \\
\text { - Capital social } \\
\text { - Capital de innovación y } \\
\text { aprendizaje }\end{array}$ & $\begin{array}{l}\text { - Indicadores de procesos } \\
\text { dinámicos }\end{array}$ \\
\hline $\begin{array}{l}\text { Balanced Business Scorecard } \\
\text { (Kaplan y Norton, 1992-1996) }\end{array}$ & $\begin{array}{l}\text { - Perspectiva financiera } \\
\text { - Perspectiva de clientes } \\
\text { - Perspectiva de procesos } \\
\text { internos } \\
\text { - Perspectiva de aprendizaje y } \\
\text { crecimiento }\end{array}$ & $\begin{array}{l}\text { - Indicadores sobre tangibles } \\
\text { - Indicadores financieros }\end{array}$ \\
\hline Dow Chemical Model (1994) & $\begin{array}{l}\text { - Capital humano } \\
\text { - Capital organizativo } \\
\text { - Capital clientes } \\
\end{array}$ & $\begin{array}{l}\text { - Indicadores de intangibles con } \\
\text { impacto sobre los resultados } \\
\text { organizativos }\end{array}$ \\
\hline $\begin{array}{l}\text { Technology Broker (Brooking, } \\
\text { 1996) }\end{array}$ & $\begin{array}{l}\text { - Activos de mercado } \\
\text { - Activos humanos } \\
\text { - Activos de propiedad } \\
\text { intelectual } \\
\text { - Activos de infraestructura }\end{array}$ & $\begin{array}{l}\text { - Indicadores cualitativos } \\
\text { - Auditoría de capital intelectual }\end{array}$ \\
\hline $\begin{array}{l}\text { Canadian Imperial Bank of } \\
\text { Comerce (Saint-Onge, 1996) }\end{array}$ & $\begin{array}{l}\text { - Organización inteligente } \\
\text { - Capital de conocimiento }\end{array}$ & - Indicadores de aprendizaje \\
\hline
\end{tabular}




\begin{tabular}{|c|c|c|}
\hline $\begin{array}{l}\text { Interested Agents Theory } \\
\text { (Atkinson, Waterhouse y } \\
\text { Wells, } \\
\text { 1997) }\end{array}$ & $\begin{array}{l}\text { - Empleados } \\
\text { - Clientes } \\
\text { - Proveedores } \\
\text { - Comunidad }\end{array}$ & - Indicadores de eficiencia \\
\hline $\begin{array}{l}\text { I } \\
\text { ntellectual Capital Index } \\
\text { (Dragonetti y Roos, 1998) }\end{array}$ & $\begin{array}{l}\text { - Capital humano } \\
\text { - Capital organizativo } \\
\text { - Capital de desarrollo y } \\
\text { renovación }\end{array}$ & - Índice de capital intelectual \\
\hline Stewart's Model (1997) & $\begin{array}{l}\text { - Capital humano } \\
\text { - Capital tecnológico } \\
\text { - Capital estructural } \\
\text { - Capital cliente } \\
\end{array}$ & $\begin{array}{l}\text { - Indicadores internos } \\
\text { - Indicadores de cliente }\end{array}$ \\
\hline $\begin{array}{l}\text { Modelo de Dirección } \\
\text { Estratégica } \\
\text { por Competencias (Bueno, } \\
\text { 1998) }\end{array}$ & $\begin{array}{l}\text { - Capital humano } \\
\text { - Capital organizativo } \\
\text { - Capital tecnológico } \\
\text { - Capital relacional }\end{array}$ & $\begin{array}{l}\text { - Indicadores de competencias } \\
\text { básicas distintivas }\end{array}$ \\
\hline $\begin{array}{l}\text { Organizacional Learning } \\
\text { Model } \\
\text { (KPMG, 1998) }\end{array}$ & $\begin{array}{l}\text { - Interacción de cultura, } \\
\text { liderazgo, } \\
\text { procesos de aprendizaje, } \\
\text { actitudes } \\
\text { humanas }\end{array}$ & $\begin{array}{l}\text { - Factores de aprendizaje } \\
\text { - Factores condicionales } \\
\text { relacionados con resultados de } \\
\text { aprendizaje }\end{array}$ \\
\hline $\begin{array}{l}\text { Knowledge Management } \\
\text { Model } \\
\text { (Arthur Andersen, 1999) }\end{array}$ & $\begin{array}{l}\text { - Perspectiva individual } \\
\text { - Perspetiva organizativa }\end{array}$ & $\begin{array}{l}\text { - Flujos de información con } \\
\text { valor } \\
\text { Añadido }\end{array}$ \\
\hline ICBS (Viedma, 2000) & $\begin{array}{l}\text { - Modelo de excelencia } \\
\text { - Benchmarking competitivo } \\
\text { - Benchmarking de } \\
\text { competencias }\end{array}$ & $\begin{array}{l}\text { - Indicadores de benchmarking } \\
\text { relacionado con las } \\
\text { competencias } \\
\text { básicas }\end{array}$ \\
\hline
\end{tabular}

(Fuente: IADE-CIC, 2003)

La denominada gestión del conocimiento constituye el punto de partida, Bontis, Chong y Richardson (2000) consideran tres componentes del capital intelectual: el capital bumano, definido como "el stock de conocimiento individual que se encuentra en los empleados de una organización", el capital estructural, que incluye el almacén no humano de conocimiento en la organización, y comprende las bases de datos, mapas conceptuales, manuales de procesos, estrategias, rutinas y todo aquello cuyo valor para la empresa es superior al de su coste material, y por último, el capital relacional, que engloba el conocimiento implícito en todas las relaciones que una organización mantiene con sus clientes, competidores, proveedores, asociaciones externas o con la administración pública 
A lo anterior se añade que el capital intelectual por tanto, puede y debe ser considerado una herramienta de gestión corporativa, en el que la creación de un capital relacional resulta clave en la construcción de la reputación organizativa y se constituiría en una especie de "capital inmaterial"

A través de la gestión del capital relacional se aumenta la posibilidad de creación de: a) capacidad relacional que es una competencia empresarial que indicaría la mayor o menor propensión a establecer y mantener relaciones duraderas con los actores del entorno de la empresa, resultante de la acción de otras variables como la personalidad del líder, las experiencias pasadas o los objetivos futuros; b) reputación corporativa un activo intangible con beneficios en múltiples áreas, entre ellas la mayor o menor posibilidad de ser considerado un socio de negocios deseable; c) normas relacionales las cuales se desarrollan y arraigan en las relaciones, a veces en forma inconsciente y sin que existan documentos escritos que las hagan explícitas, y son determinantes para el desarrollo armónico del proceso relacional, son expectativas acerca del comportamiento mutuo; d) riesgo relacional es la probabilidad y consecuencia de no tener una cooperación satisfactoria, o de un comportamiento oportunista del socio; por último, e) los activos relacionales que no pertenecen a una ni a otra parte sino a la relación en sí misma; ellos realizan una contribución importante a los procesos de creación de valor corporativo.

En función de todo ello, el desarrollo adecuado de los anteriores cinco ítems, haría posible obtener rentas relacionales, entendidas como "beneficios superiores generados conjuntamente por la relación de intercambio que no pueden ser generados por ninguna de las empresas de manera aislada y que sólo pueden crearse mediante la unión de las contribuciones conjuntas de los dos socios de la alianza" (Dyer y Singh, 1998)

Conseguir incrementar la competitividad de las organizaciones afianzando el capital relacional valida la relación positiva entre el capital intelectual, la generación de riqueza y el crecimiento económico. De este modo el papel de las redes establecidas con los stakeholders desempeña un papel fundamental en los activos intangibles.

La literatura muestra que en la experiencia, algunos aprendizajes de los empresarios se han derivado de actividades con utilidad potencial en la creación de este activo intangible como: asistencia a ruedas de negocios, contacto directo, referenciación de otros clientes, alianzas del sector, relación con instituciones de promoción, relaciones informales, cultura de asociatividad.

Volver costumbre la dinámica empresarial de la búsqueda del capital relacional, mediante la coordinación tácita de estrategias, permite movimientos competitivos que llevarán a la necesidad de incorporar a los intangibles como fuente de generación de efectivo en la información contable. Este efecto del comportamiento estratégico es una pieza clave en el funcionamiento de una organización, obviamente las consecuencias 
potenciales contribuyen a la estabilidad de las relaciones existentes y a minimizar un impacto negativo en la disolución de una relación.

En momentos donde la incertidumbre y volatilidad reina en los mercados por los efectos de la globalización, la teoría del capital relacional, caracterizada por ser el capital que la empresa genera a partir de su relación con el entorno, predice un elemento de mayor rivalidad entre compañías similares, al llevarlas a incorporar conocimientos como consecuencias del valor derivado de sus relaciones con los agentes de mercado y la sociedad en general.

Como corolario, el capital relacional definido como la habilidad de la empresa para interactuar positivamente con el entorno, estimula el potencial de creación de riqueza al fortalecer el capital humano y el capital estructural y destaca el papel de la innovación en función del conocimiento externo de relaciones organizativas que la empresa tiene.

Por tanto según las argumentaciones presentadas, se subraya el reconocimiento al capital relacional como un conjunto de activos intangibles generadores de oportunidades de negocio en espera de administración del know how adquirido, de tal forma que sustente la reacción del conocimiento y la innovación, soportados en el crecimiento de los negocios y por ende en el incremento del capital.

\section{ENFOQUE DE EVALUACIÓN}

La gestión de activos intangibles, de acuerdo a sus características, exige sistemas de dirección estratégica en toda la organización, cuya puesta en marcha permitan la potenciación del valor percibido en el mercado, con repercusiones positivas en la valoración de la empresa.

Muy seguramente si se quiere avanzar más allá de una descripción cualitativa, se debe elaborar cierta medida cuantitativa de los factores claves a considerar en el capital relacional. En realidad, esto se ha hecho en algunos estudios, por ejemplo Macagnan (2007); Rodríguez (2006); Hormiga, et al. (2005); Suárez, et al. (2005) y Pacheco (2004).

Para analizar la gestión del capital relacional con el objetivo de otorgar más valor a la compañía basado en capitales intangibles, se adelantan diversos ejercicios; entre otros, se podrían analizar 44 factores representativos de 8 dimensiones con el objeto de determinar globalmente la perspectiva sistémica de aspectos, comportamientos y actitudes relacionales. 
DIMENSIÓN: CLIENTES

- Cantidad, diversidad, calidad

- Lealtad, fidelización

- Satisfacción

- Contacto, procesos de comunicación

- Estrategias de venta y postventa

- Medidas de satisfacción

- Capacidad de respuesta

- Tiempo invertido en establecer relaciones con clientes

- Grado de internacionalización

\section{DIMENSIÓN: PROVEEDORES}

- Red de proveedores

- Evolución de precios

- Logística

- Localización

DIMENSIÓN: EMPLEADOS

- Compromiso

- Motivación

- Relación contractual

- Productividad

\section{DIMENSIÓN: SOCIOS /DUEÑOS}

- Compromiso

- Publicación de acciones ambientales, sociales

- Relación con socios, accionistas y empleados

- Rentabilidad

\section{DIMENSIÓN: COMPETENCIA}

- Conocimiento

- Experiencia

- Especialización

- Know who 


\section{DIMENSIÓN: SOCIEDAD}

- Años de constitución de la empresa

- Conflicto de intereses

- Revelación del capital estructural-tecnológico

- Apertura de nuevos mercados

- ISO o similar

\section{DIMENSIÓN: PARTICIPACIÓN EN REDES}

- Apoyo de relaciones informales

- Vinculación con relaciones formales

- Contactos dentro del sector

- Alianzas estratégicas

- Participación en eventos

- Conectividad

- Centros de innovación

\section{DIMENSIÓN: REPUTACIÓN}

- Prestigio del Empresario

- Imagen de la Organización

- Confianza

- Colaboraciones y alianzas

- Lugar estratégico

- Stakeholders

- Proyectos sociales, ambientales

Hemos recorrido un buen camino en tratar de entender, como una rica mezcla de activos intangibles, permite avanzar con mayor velocidad en la extensión del horizonte de oportunidades de negocios, situación clave para obtener resultados esperados en la formación de capital.

\section{CONCLUSIONES}

El papel del capital relacional en las organizaciones, descansa en el valor que tiene para una empresa el conjunto de relaciones que mantiene con el exterior, sin lugar a duda clave potencial de éxito para generar negocios en el futuro, a partir de su relación con los agentes del entorno. En este sentido, las organizaciones que reconocen la 
importancia de capturar este conocimiento, despiertan interés por tener una adecuada sistematización, comprender las particularidades de los actores del entorno, desplegar estrategias, examinar relación entre variables; aspectos informativos que permitan crear vínculos que generen una ventaja competitiva.

Uno de los grandes retos en el diseño de un modelo aplicado de capital relacional, señala la urgencia de una dirección inteligente que al entender el valor de administrar bienes de carácter intangible, ofrezca matices diferenciadores que proporcionen ventajas a la empresa sobre otras, para así crear oportunidades de negocio que generen riqueza y al final se reflejen en la cuenta de resultados.

Son numerosos los estudios en los que constatan la importancia estratégica de esa relación intrínseca entre el capital relacional y la posición de mercado, la cual permite lograr oportunidades de negocio como respuesta a la lealtad, la confianza, el compromiso, la reputación, los intercambios relacionales exitosos, la disposición comportamental y la comunicación con el entorno y el mercado.

No obstante la importancia que tiene estos activos intangibles dentro del conocimiento organizacional, la eficiencia en la asignación cuantitativa dentro de los estados financieros dista de la valoración de la organización representada por el valor de los activos de la información y el conocimiento en el balance. Una reflexión posterior, la constituiría un estudio de los pasivos intangibles, los cuales al igual que los activos intangibles afectan la rentabilidad de la empresa.

El potencial de reconocer la capacidad de gestionar el capital relacional, es parte de una serie de iniciativas diseñadas para ayudar a las empresas a responder a los desafíos sobre el reconocimiento contable de los activos intangibles.

\section{REFERENCIAS}

- BENAVIDES, C. et al. (2003). Gestión del conocimiento y calidad total. Madrid: Díaz de Santos.

- BONTIS, N., et. al. (2000): Intellectual capital and business performance in Malaysian industries. Journal of Intellectual Capital, vol. 1, n.1.

- BROOKING, A. (1997). El capital intelectual, el principal activo de las empresas del tercer milenio. Barcelona: Paidós Ibérica.

- BUENO, E. (2002). El capital social en el nuevo enfoque del capital intelectual de las organizaciones. Revista de psicología del trabajo y de las organizaciones.

- DYER, J.H. y SINGH, H. (1998): "The Relational View: Cooperative Strategy and Sources of Interorganizational Competitive Advantage". Academy of Management Review.

- EDVINSSON, L. MALONE, M. (1998). El capital intelectual. Cómo identificar y calcular el valor de los recursos intangibles de su empresa. Barcelona: Gestión 2000. 
- EUROFORUM ESCORIAL (1998). Medición del capital intelectual: modelo Intelect. Madrid: Euroforum Escorial.

- IADE-CIC (2003): “Modelo Intellectus: Medición y gestión del capital intelectual", Documento Intellectus $n^{\circ} 5$.

- ONGE, H. (1996) "Tacit knowledge: the key to the strategic alignment of intelectual capital, Strategy \& Leadershyp" Vol. 24, no 2.

- PALACIOS, D. (2002). La creación de competencias distintivas dinámicas mediante la gestión del conocimiento y su efecto sobre el desempeño: análisis empirico en las industrias de biotecnología y telecomunicaciones. Tesis doctoral, Universitat Jaume I, Castellón.

- PAlOmO, M. (2003) La evaluación de activos intangibles. Parte I. Los modelos. Vol. VI. No. 20.

- REQUENA, Félix (1991). Redes sociales y mercado de trabajo. Elementos para una teoría del capital relacional. Madrid, Centro de Investigaciones Sociológicas y Siglo XXI. Monografías, No 19.

- SVEIBY, K.E. (1997). “The intangible assets monitor". Journal of Human Resource Costing \& Accounting.

- SWART, J. (2006): "Intellectual Capital: Disentangling an Enigmatic Concept". Journal of Intellectual Capital, $\mathrm{n}^{\circ} 7$.

- VIEDMA, J.M., et. al. (2004). La gestión del capital intelectual en Mataró (GCIM). En Medición, controly gestión de los intangibles. Barcelona: Deusto y ACCID. 